Editorial

\title{
Editorial: Video Games as Demanding Technologies
}

\author{
Nicholas David Bowman \\ College of Media and Communication, Texas Tech University, Lubbock, TX 79410, USA; E-Mail: nick.bowman@ttu.edu
}

Submitted: 2 December 2019 | Published: 20 December 2019

\begin{abstract}
From the middle-20th century to today, video games have grown from an idiosyncratic interest of computer programmers and engineers to a globally dominant form of media entertainment. Advances in technology and creativity have combined to present players with interactive experience that vary in their cognitive, emotional, physical, and social complexity. That video games constitute co-authored experiences-dialogues between the player and the system-is at least one explanation for their appeal, but this co-authorship brings with it an enhanced set of requirements for the player's attention. For this thematic issue, researchers were invited to debate and examine the cognitive, emotional, physical, and social demands of video games; their work (as well as the impetus for this work) is summarized below.
\end{abstract}

\section{Keywords}

interactivity-as-demand; media history; media psychology; video games

\section{Issue}

This editorial is part of the issue "Video Games as Demanding Technologies" edited by Nicholas David Bowman (Texas Tech University, USA).

(C) 2019 by the author; licensee Cogitatio (Lisbon, Portugal). This article is licensed under a Creative Commons Attribution 4.0 International License (CC BY).

\section{Introduction}

It could be said that a driving motivation behind the development of mediated technologies is to close myriad gaps between users and spaces, events, and agents (or some combination of each). Ancient stones allowed governments to communicate rules and regulations throughout their domains of influence (such as the Rosetta Stone; Bierbrier, 1999). Written and printed texts provided (depending on the medium, variably) permanent access to the stories and values of far-away and bygone generations (much to the chagrin of Socrates, who fears that mediation would remove the author from their work; Plato as translated by Jowett, 1999). Advances in broadcasting technologies (such as radio and television) allowed at-home audiences to follow their favorite sporting events happening hundreds of miles away, and social media allowing for nearly unfettered access to the athletes themselves (Bowman, 2013). In closing these gaps, emerging media technologies follow a similar pattern of evolution: First improving on existing media before emerging as (relatively speaking) "new" media of their own (Stober, 2004). To highlight one example, the earliest films began as technological demonstrations of "pic- tures in motion" (Leslie, 2001) before moving towards basic recordings of standard Vaudeville and other singlestage theatre performance. Decades would pass until film directors understood how to splice from several different film rolls (each recording their own scenes) to tell more compelling stories unique to the film medium: the theory of montage in film commonplace in modern cinema (Harrah, 1954).

The earliest video game technologies also began as technological demonstrations, largely designed as "interactive television" systems (U.S. Patent No. 2,455,992, 1948) in which users could exercise a degree of control over the form and content of what was portrayed onscreen: User input had a direct impact on on-screen output (Steuer, 1992). However, early game development quickly focused more on "interactive" than "television" and by the middle of the 20th century, video game designed operated on three principles: to tax computational resources; to generate a unique experience for each run; and to actively involve users in a pleasurable way (Graetz, 1981). By the start of the 21st century, video games had overtaken films and television (and other screen-based media) as the most profitable form of entertainment media. As described by Meier (2012), good 
video games provide players with "a series of interesting decisions."

Here, video games break from contemporary film and television in that they shift authorial control to the players, or at least invite players to co-author variably unique experiences (Wellenreiter, 2015), and these pleasures of control are generally assumed to explain the popularity of video games (Grodal, 2000). Conversely, Bowman (2016) argued that although this authorial control is a potentially powerful source of entertainment, it could also be viewed as requirements-demands foisted on users that might not always be so well-received or, at least, not universally so. At least four sources of demand were proposed in Bowman (2016), refined in Bowman (2018), and examined empirically in Bowman, Wasserman, and Banks (2018): cognitive demands associated with understanding in-game challenges; emotional demands associated with investment into the game's unfolding narrative; physical demands associated with fine and gross motor control of the game's controls; and social demand associated with variable social relations with in-game characters and other players. From a nascent interactivity-as-demand model, these (at least) four sources of demand are proposed as critical mediators of interactive media content and their resultant effects (Figure 1).

In response to these notions, and using Bowman (2018) as animus, nearly three-dozen authorship teams submitted manuscript abstracts for potential inclusion in this thematic issue, and through several rounds of editor and peer review, nine manuscripts were retained for publication. Those manuscripts expanded demand concepts into empirical observations, critiqued conceptual shortcomings in nascent scholarship, and directly applied demand notions into models of game design.

\section{Contributions to the Thematic Issue}

Several of the manuscripts in this thematic issue expanded on one or more of the demand concepts. For example, in "Modality-Specific Effects of Perceptual Load in Multimedia Processing," Fisher and colleagues (2019) demonstrate the importance of a more nuanced approach to cognitive demands informed from a neural perspective, cleaving perceptual load (attentional demands placed on sensory systems, such as hearing and sight) from cognitive load (processing demands which involve higher-order systems, such as working memory; see Fisher, Huskey, Keene, \& Weber, 2018). Their key finding suggested that while increased cognitive loads (more aligned with how cognitive demand was explicated in Bowman, 2018) depleted attentional resources in both auditory and visual channels, the influence of perceptual load was modality-dependent (in this study, restricted to visual systems). Given the multimodal nature of video games, Fisher et al.'s (2019) work speaks to the criticality of examining how gamers both perceive and process information. As an added bonus, this article-or rather, the proposed study-was also preregistered, with study predictions, materials, and data analysis files freely available at https://osf.io/as2u5.

Likewise, the work of Large and colleagues (2019), "Cognitive and Behavioral Correlates of Achievement in a Complex Multi-Player Video Game," found that experienced layers of the multiplayer online battle arena game League of Legends exhibited superior performance at a number of cognitive demand tasks that could be understood both in terms of perceptual load (speed of processing and object tracking tasks, among others) as well as cognitive load (deductive reasoning and a variety of learning tasks, among others). Both articles demon-

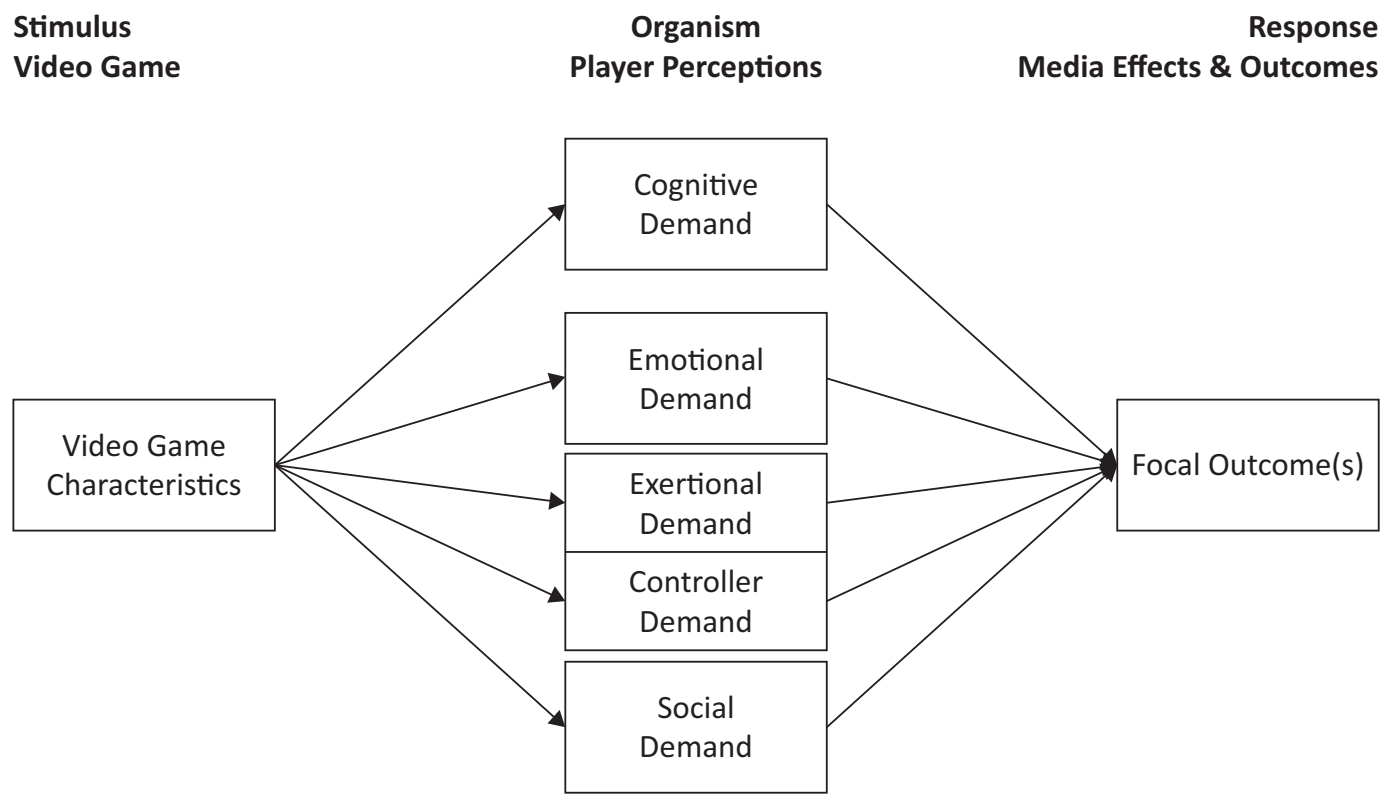

Figure 1. A conceptual model of interactivity-as-demand. 
strate the importance of using behavioral and physiological measures to better understand and refine constructs, such as augmenting the self-report measure of cognitive demand reported in Bowman et al. (2018) that might have unnecessarily forwarded a definition focused on recollections of deliberative thought.

Of course, cognitive demand can still be directly tied to in-game challenges and difficulties, as demonstrated by Wulf and colleagues' (2019) "Harder, Better, Faster, Stronger? The Relationship between Cognitive Task Demands in Video Games and Recovery Experiences." In their work, playing the video game Tetris facilitated feelings of mastery and psychological detachment following an experimental induction designed to induce mild levels of fatigue-these effects were found regardless of how cognitively demanding (re: difficult) the game was. However, higher levels of cognitive demand diminished players' sense of control following the game, suggesting that the mental effort required of the harder game likely had a fatiguing effect on players (similar findings reported in Bowman \& Tamborini, 2015). This article also made study materials and data analysis freely available at https://osf.io/jgp58, and the research provides additional data points for the broader literature focused on interactive media and psychological outcomes associated with well-being.

Three other manuscripts applied elements of the interactivity-as-demand to better understand critical issues in game studies associated with gender studies, trolling behaviors, and moral decision-making. Droumeva's (2019) "Audible Efforts: Gender and Battle Cries in Classic Arcade Fighting Games" used demands as a framework for understanding the gendered battle cries during gameplay-from her abstract, no-screen characters "constantly making sounds of strife, struggle, or victory while conducting surrogate labor for [the players]" (2019, p. 186). Battle cries are framed as reflective of physical and emotional demands, and her analysis reveals that these cries are decidedly gendered and trending such that female characters' cries have become louder and more intensely sexualized over time. Cook's (2019) "Between a Troll and a Hard Place: The Demand Model's Answer to One of Gaming's Biggest Problems" proposes that competing demands, both between a player and their game, and players and each other, might explain why some players engage in antisocial trolling behaviors. Put simply, trolling might be a maladaptive behavior that results from either: (a) Bored (under-demanded) or frustrated (over-demanded) players; or (b) is the consequence of balancing emotional and social demands of playing with others. Hodge and colleagues' (2019) “(A)morally Demanding Game? The Results and Implications of Moral Decision-Making in a Purpose Made Game" continues a line of research into gaming and morality with somewhat surprising findings - they failed to replicate prior work showing that gamers' "real world" and chronically accessible moral intuitions are predictive of observed in-game decisions
(Joeckel, Bowman, \& Dogruel, 2012; Tamborini et al., 2016). One suggestion offered by Hodge et al. (2019), informed in part by the observed time-lag for in-game decisions (four to six seconds, on average), is that players engaged in more deliberative and cognitively demanding decision-making; in past work, such decisions were more intuitive and based on what we might understand as emotional reactions. To this end, future work into moral decision-making might benefit from a dual focus on cognitive and emotional demands.

Two other manuscripts offered more direct constructive criticisms of interactivity-as-demand, one challenging a myopic view on social demand, and the other suggesting a focus on gaming infrastructures. Deterding's (2019) "Interaction Tension: A Sociological Model of Attention and Emotion Demands in Video Gaming" provides re-analysis of qualitative interviews with 19 German-speaking gamers and those in the gaming industry (transcripts and data analysis files available at https://osf.io/3w4js; transcripts in German). From this data, two critical trends are presented to game scholars: (1) Social demands cannot be treated as orthogonal from other sources of demand (such as cognitive and emotional demands); and (2) social demands are felt in advance of any exposure to gaming content and/or other gamers, given the social norms that surround video games. Mirowski and Harper's (2019) "Elements of Infrastructure Demand in Multiplayer Video Games" provides a novel operationalization of infrastructure demands (here, specific to online multiplayer games) guided by Star (1999). Through an analysis of the mundane and often invisible components of the macro-level systems that gird and support online gaming-using Blizzard Entertainment's Overwatch League as an example-the authors present a compelling case for how gaming infrastructures likely and jointly impact player experiences.

Finally, the authorship team of Agius and DaylamaniZad (2019) break from their peers in the eight manuscripts summarized above by incorporating an interactivity-as-demand approach into a more comprehensive model of game design. In their work, "Reducing Extrinsic Burdens on Players of Digital Games: An Integrated Framework" (2019), the authors align rather strongly with the earliest arguments on cocreation put forth in Bowman (2016), suggesting that when games over-demand of players, they become burdensome. Their work explores gaming demands in terms of their interfaces, their interactions, and core gameplay mechanics, and they offer specific considerations at each later than can be taken by designers in order to lower unwanted demands on players.

Taken as a whole, these nine works both challenge and extend on the basic ideas presented in Bowman (2018), and collectively demonstrate how a more nuanced consideration of the numerous demands of interactive media-individually and jointly - can improve how we understand the medium's past, present, and future development. It was a pleasure and an honor to read and 
review each one, and I am eager to see the research inspired by the works presented herein.

\section{Acknowledgments}

I would like to acknowledge the authors for this thematic issue as well as the nearly three dozen manuscript reviewers, in addition to the authorship teams who submitted over 30 different abstracts for potential inclusion in this work. Jaime Banks (Texas Tech University, USA) was instrumental in providing top-level feedback for this editorial and other editorial decisions, as was Kevin Koban (Technological University at Chemnitz, Germany). I am also indebted to the many faculty and students in the TTU College of Media and Communication Research Nexus, for their ongoing support of this research.

\section{Conflict of Interests}

The author declares no conflict of interests.

\section{References}

Agius, H., \& Daylamani-Zad, D. (2019). Reducing extrinsic burdens on players of digital games: An integrated framework. Media and Communication, 7(4), 247-259.

Bierbrier, M. L. (1999). The acquisition by the British Museum of antiquities discovered during the French invasion of Egypt. In W. V. Davies \& T. G. H. James (Eds.), Studies in Egyptian antiquities (pp. 111-114). London: British Museum Publications.

Bowman, N. D. (2013). Social media, spaghetti westerns, and modern spectator sports. In D. Coombs \& B. Batchelor (Eds.), American history through American sports (Vol. 3, pp. 31-48). Santa Barbara, CA: Praeger.

Bowman, N. D. (2016). Video gaming as co-production. In R. Lind (Ed.), Produsing 2.0: The intersection of audiences and production in a digital world (Vol. 2, pp. 107-123). New York, NY: Peter Lang Publishing.

Bowman, N. D. (2018). The demanding nature of video game play. In N. D. Bowman (Ed.), Video games: A medium that demands our attention (pp. 1-24). New York, NY: Routledge.

Bowman, N. D., \& Tamborini, R. (2015). "In the mood to game": Selective exposure and mood management processes in computer game play. New Media \& Society, 17(3), 375-393. https://doi.org/10.1177/ 1461444813504274

Bowman, N. D., Wasserman, J., \& Banks, J. (2018). Development of the video game demand scale. In $\mathrm{N}$. D. Bowman (Ed.), Video games: A medium that demands our attention (pp. 208-233). New York, NY: Routledge.

Cook, C. L. (2019). Between a troll and a hard place: The demand model's answer to one of gaming's biggest problems. Media and Communication, 7(4), 176-185.
Deterding, S. (2019). Interaction tension: A sociological model of attention and emotion demands in video gaming. Media and Communication, 7(4), 226-236.

Droumeva, M. (2019). Audible efforts: Gender and battle cries in classic arcade fighting games. Media and Communication, 7(4), 186-197.

Fisher, J. T., Hopp, F. R., \& Weber, R. (2019). Modalityspecific effects of perceptual load in multimedia processing. Media and Communication, 7(4), 149-165.

Fisher, J. T., Huskey, R., Keene, J. R., \& Weber, R. (2018). The limited capacity model of motivated mediated message processing: Looking to the future. Annals of the International Communication Association, 42(4), 291-315. https://doi.org/10.1080/23808985. 2018.1534551

Goldsmith, J. T., Ray, M. E. (1948). U.S. Patent No. $2,455,992$. Washington, DC: U.S. Patent and Trademark Office. Retrieved from https://patents.google. com/patent/US2455992A/en

Graetz, J. M. (1981). The origin of spacewar. Creative Computing, 7(8), 56-67. Retrieved from http://www.wheels.org/spacewar/creative/ SpacewarOrigin.html

Grodal, T. (2000). Video games and the pleasures of control. In D. Zillmann \& P. Vorderer (Eds.), Media entertainment: The psychology of its appeal (pp. 197-213). Mahwah, NJ: LEA.

Harrah, D. (1954). Aesthetics of the film: The PudovkinArnheim-Eisenstein theory. The Journal of Aesthetics and Art Criticism, 13(2), 163-174. https://doi.org/ 10.2307/425910

Hodge, S. E., Taylor, J., \& McAlaney, J. (2019). (A)morally demanding game? The results and implications of moral decision-making in a purpose made game. Media and Communication, 7(4), 213-225.

Joeckel, S., Bowman, N. D., \& Dogruel, L. (2012). Gut or game: The influence of moral intuitions on decisions in virtual environments. Media Psychology, 15(4), 460-485. https://doi.org/10.1080/15213269. 2012.727218

Large, A. M., Bediou, B., Cekic, S., Hart, Y., Bavelier, D., \& Green, C. S. (2019). Cognitive and behavioral correlates of achievement in a complex multi-player video game. Media and Communication, 7(4), 198-212.

Leslie, M. (2001). The man who stopped time. Stanford Magazine. Retrieved from https://stanfordmag.org/ contents/the-man-who-stopped-time/

Meier, S. (2012). Interesting decisions. Paper presented at the Game Developers Conference, San Francisco, California, USA. Retrieved from https://www. gdcvault.com/play/1015756/Interesting

Mirowski, A., \& Harper, B. (2019). Elements of infrastructure demand in multiplayer video game. Media and Communication, 7(4), 237-246.

Plato. (1999). Phaedrus (B. Jowett, Trans.). Salt Lake City, UT: Project Gutenberg. Retrieved from http://www. gutenberg.org/files/1636/1636.txt

Star, S. L. (1999). The ethnography of infrastructure. 
American Behavioral Scientist, 43(3), 377-391. https://doi.org/10.1177/00027649921955326

Steuer, J. (1992). Defining virtual reality: Dimensions determining telepresence. Journal of Communication, 42(4), 73-93. https://doi.org/10.1111/j.1460-2466. 1992.tb00812.x

Stober, R. (2004). What media evolution is: A theoretical approach to the history of new media. European Journal of Communication, 19(4), 483-505. https:// doi.org/10.1177/0267323104049461

Tamborini, R., Prabhu, S., Bowman, N. D., Hahn, L., Klebig, B., Grall, C., \& Novotny, E. (2016). The effect of moral intuitions on decisions in video-game play: Tempo- rary and chronic intuition accessibility. New Media \& Society, 20(2), 564-580. https://doi.org/10.1177/ 1461444816664356

Wellenreiter, M. (2015). Screenwriting and authorial control in narrative video games. Journal of Screenwriting, 6(1), 343-361. https://doi.org/10.1386/josc.6.3. 343_1

Wulf, T., Rieger, D., Kümpel, A. S., \& Reinecke, L. (2019). Harder, better, faster, stronger? The relationship between cognitive task demands in video games and recovery experiences. Media and Communication, 7(4), 166-175.

\section{About the Author}

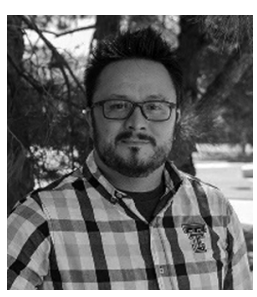

Nicholas David Bowman (PhD, Michigan State University) is Associate Professor of Journalism and Creative Media Industries at Texas Tech University, USA, and Fulbright-Wu Jing-Jyi Arts and Culture Fellow at the National Chengchi University, Taiwan. His research examines the cognitive, emotional, social, and physical demands of interactive media, and he has published over 80 peer-reviewed manuscripts focused on media psychology and related issues. He was most recently editor of Communication Research Reports and is an associate editor with Journal of Media Psychology. 